\title{
Automated Synthesis of a Protected $N$-linked Glycoprotein Core Pentasaccharide
}

\author{
Daniel M. Ratner, Erika R. Swanson and Peter H. Seeberger ${ }^{\mathrm{J} *}$ \\ Dept. of Chemistry, Massachusetts Institute of Technology \\ seeberger@org.chem.ethz.ch
}

\section{Supplementary Material}

General Methods. All chemicals used were reagent grade and used as supplied except where noted. Trimethylsilyl trifluoromethanesulfonate (TMSOTf) was purchased from Acros Chemicals. Dichloromethane $\left(\mathrm{CH}_{2} \mathrm{Cl}_{2}\right)$, diethyl ether $\left(\mathrm{Et}_{2} \mathrm{O}\right)$, and tetrahydrofuran (THF) were purchased from J.T. Baker (Cycletainer ${ }^{\mathrm{TM}}$ ) and passed through neutral alumina columns prior to use. Toluene was purchased from J.T. Baker (Cycletainer ${ }^{\mathrm{TM}}$ ) and passed through a neutral alumina column and a copper (II) oxide column prior to use. Pyridine and acetonitrile were refluxed over calcium hydride and distilled prior to use. Analytical thin-layer chromatography was performed on E. Merck silica column $60 \mathrm{~F}_{254}$ places $(0.25 \mathrm{~mm})$. Compounds were visualized by dipping the plates in a cerium sulfateammonium molybdate solution followed by heating. Liquid column chromatography was performed using forced flow of the indicated solvent on Silicycle 230-400 mesh (60 $\AA$ A pore diameter) silica gel. Optical rotation was recorded on a Perkin-Elmer 241 polarimeter using a sodium lamp $(589 \square)$ at $24^{\circ} \mathrm{C}$. IR spectra were obtained on a PerkinElmer System 2000 series FTIR spectrometer. ${ }^{1} \mathrm{H}$ NMR spectra were obtained on a Bruker (400 MHz) or a Varian VXR-500 (500 MHz) and are reported in parts per million (D) relative to $\mathrm{CHCl}_{3}(7.27 \mathrm{ppm})$. Coupling constants $(J)$ are reported in Hertz. ${ }^{13} \mathrm{C} \mathrm{NMR}$ 
spectra were obtained on a Bruker (100 MHz) or a Varian VXR-500 (125 MHz) and are reported in $\square$ relative to $\mathrm{CDCl}_{3}(77.23 \mathrm{ppm})$ as an internal reference. High-resolution mass spectrometry was performed on a Bruker DALTONICS APEX II, 3 Tesla, FT-ICRMS.

\section{tert-B utyld imeth y lsilyl 3,6-d i-O -benzyl-2-deoxy-2-trichloroacetimido- $\square-D-$}

glucopyranoside 6. Differentially protected glucosamine $\mathbf{5}^{11}(5.5 \mathrm{~g}, 8.9 \mathrm{mmol})$ was dissolved in $\mathrm{CH}_{2} \mathrm{Cl}_{2}(60 \mathrm{~mL})$ and cooled to $0^{\circ} \mathrm{C}$. Triethylsilane $(8.5 \mathrm{~mL}, 53.5 \mathrm{mmol})$ was added and the resulting mixture was stirred for $10 \mathrm{~min}$. Trifluoromethanesulfonic acid $(3.4 \mathrm{~mL}, 44.5 \mathrm{mmol})$ and trifluoromethanesulfonic acid anhydride $(1.3 \mathrm{~mL}, 8.9$ mmol) were added simultaneously to the cooled solution and the mixture was stirred at $0^{\circ} \mathrm{C}$ for $30 \mathrm{~min}$. The solution was warmed slowly to room temperature over a period of 1 h. The reaction mixture was poured into a saturated aqueous solution of $\mathrm{Na}_{2} \mathrm{CO}_{3}$. The aqueous layer was extracted with $\mathrm{CH}_{2} \mathrm{Cl}_{2}(2 \times 20 \mathrm{~mL})$ and the organic layer was dried over $\mathrm{NaSO}_{4}$, filtered, and solvents removed in vacuo. Purification by flash silica column chromatography (10\% 25\% EtOAc/hexanes) afforded 6 as an oil (3.47 g, 63\% yield). $[\square]^{24}{ }_{\mathrm{D}}:-13.4^{\circ}$ (c 1.8, $\mathrm{CH}_{2} \mathrm{Cl}_{2}$ ); IR (thin film) 2929, 2858, 1692, 1529, 1070, $838 \mathrm{~cm}^{-1} ;{ }^{1} \mathrm{H}$ NMR (400 MHz, $\left.\mathrm{CDCl}_{3}\right) \square 7.40-7.27(\mathrm{~m}, 10 \mathrm{H}), 6.99(\mathrm{~d}, J=8.1 \mathrm{~Hz}, 1 \mathrm{H}), 5.05(\mathrm{~d}, J=7.8$ $\mathrm{Hz}, 1 \mathrm{H}), 4.83-4.76(\mathrm{~m}, 2 \mathrm{H}), 4.64-4.57(\mathrm{~m}, 2 \mathrm{H}), 3.95(\mathrm{dd}, J=8.6,10.5 \mathrm{~Hz}, 1 \mathrm{H}), 3.76-3.71$ $(\mathrm{m}, 3 \mathrm{H}), 3.62-3.55(\mathrm{~m}, 2 \mathrm{H}), 2.92(\mathrm{~s}, 1 \mathrm{H}), 0.92(\mathrm{~s}, 9 \mathrm{H}), 0.16(\mathrm{~s}, 3 \mathrm{H}), 0.13(\mathrm{~s}, 3 \mathrm{H}) ;{ }^{13} \mathrm{C}$ NMR (400 MHz, $\left.\mathrm{CDCl}_{3}\right) \square 161.9,138.2,137.8,128.7,128.6,128.2,128.0,128.0,127.8$, $94.9,92.7,79.8,74.4,74.0,73.8,73.3,70.7,60.1,25.8,18.0,-4.0,-5.0 ;$ ESI MS $m / z\left(\mathrm{M}^{+}\right.$ $+\mathrm{Na}^{+}$) calcd 640.1426, found 640.1400. 


\section{4-O-Acetyl-3,6-di- $O$-benzyl-2-deoxy-2-trichloroacetimido-D-D-glucopyranosyl}

trichloroacetimidate 2. A solution of $6(1.06 \mathrm{~g}, 1.71 \mathrm{mmol})$ in $\mathrm{CH}_{2} \mathrm{Cl}_{2}(20 \mathrm{~mL})$ was cooled to $0^{\circ} \mathrm{C}$. Acetic anhydride $(0.24 \mathrm{~mL}, 2.57 \mathrm{mmol})$ was added and the resulting solution was stirred for $5 \mathrm{~min}$. Dimethylaminopyridine (314 $\mathrm{mg}, 2.57 \mathrm{mmol}$ ) was added and the reaction was allowed to warm slowly to room temperature while stirring overnight. The mixture was diluted with $\mathrm{CH}_{2} \mathrm{Cl}_{2}(30 \mathrm{~mL})$ and the organic layer was washed with $5 \% \mathrm{HCl}(2 \times 30 \mathrm{~mL})$. The organic layer was dried over $\mathrm{Na}_{2} \mathrm{SO}_{4}$, filtered, and solvents removed in vacuo to afford tert-butyldimethylsilyl 3,6-di-O-benzyl-2-

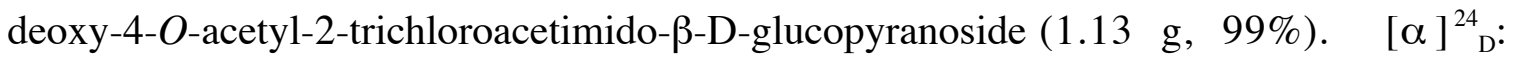
$+9.0^{\circ}\left(c 1.7, \mathrm{CH}_{2} \mathrm{Cl}_{2}\right)$; IR (thin film) 3354, 1715, 1527, 1249, $1067 \mathrm{~cm}^{-1} ;{ }^{1} \mathrm{H}$ NMR (400 $\left.\mathrm{MHz}, \mathrm{CDCl}_{3}\right) \square 7.35-7.25(\mathrm{~m}, 10 \mathrm{H}), 7.12(\mathrm{~d}, J=7.7 \mathrm{~Hz}, 1 \mathrm{H}), 5.20(\mathrm{~d}, J=7.8 \mathrm{~Hz}, 1 \mathrm{H})$, $5.09(\square \mathrm{t}, J=9.4 \mathrm{~Hz}, 1 \mathrm{H}), 4.69(\mathrm{~d}, J=11.1 \mathrm{~Hz}, 1 \mathrm{H}), 4.59(\mathrm{~d}, J=11.1 \mathrm{~Hz}, 1 \mathrm{H}), 4.54(\mathrm{~s}$, 2H), $4.29(\square \mathrm{t}, J=10.3 \mathrm{~Hz}, 3 \mathrm{H}), 3.71-3.69(\mathrm{~m}, 1 \mathrm{H}), 3.58-3.52(\mathrm{~m}, 1 \mathrm{H}), 1.89(\mathrm{~s}, 3 \mathrm{H}), 0.92$ (s, 9H), 0.18 (s, 3H), 0.15 (s, 3H); ${ }^{13} \mathrm{C}$ NMR (400 MHz, $\mathrm{CDCl}_{3}$ ) $\square$ 169.9, 161.9, 138.0, $137.8,128.6,128.4,128.0,127.8,127.8,94.3,92.7,77.4,74.1,73.6,73.4,71.8,69.8$, 60.8, 25.8, 21.0, 18.0, -4.0, -5.1; ESI MS $m / z\left(\mathrm{M}^{+}+\mathrm{Na}^{+}\right)$calcd 682.1532, found 682.1543

A solution tert-butyldimethylsilyl 3,6-di- $O$-benzyl-2-deoxy-4- $O$-acetyl-2trichloroacetimido-马-D-glucopyranoside $(1.13 \mathrm{~g}, 1.71 \mathrm{mmol})$ in THF $(18 \mathrm{~mL})$ was cooled to $0^{\circ} \mathrm{C}$. Acetic acid $(0.15 \mathrm{~mL}, 2.68 \mathrm{mmol})$ and then tetrabutylammonium fluoride $(1.0 \mathrm{M}$ in THF, $2.68 \mathrm{~mL}, 2.68 \mathrm{mmol}$ ) were added to the cooled solution. The reaction mixture was allowed to warm slowly to room temperature while stirring overnight. The reaction mixture was diluted with EtOAc and washed with $\mathrm{NaHCO}_{3}(2 \times 30 \mathrm{~mL})$ and $\mathrm{H}_{2} \mathrm{O}(1 \times 30$ 
$\mathrm{mL}$ ). The organic layer was dried over $\mathrm{Na}_{2} \mathrm{SO}_{4}$, filtered, and solvents removed in vacuo. The crude material $\left(887 \mathrm{mg}, 1.62 \mathrm{mmol}\right.$ ) was dissolved in $\mathrm{CH}_{2} \mathrm{Cl}_{2}(16 \mathrm{~mL})$ and trichloroacetonitrile (4 mL). After stirring for $5 \mathrm{~min}$, DBU (49 $\square \mathrm{L}, 0.32 \mathrm{mmol}$ ) was added and the reaction mixture was allowed to stir for $1.5 \mathrm{~h}$. The reaction mixture was passed through a silica plug, washed with EtOAc and solvents removed in vacuo. Purification by flash silica column chromatography (25\% EtOAc/hexanes) afforded 2 (942 mg, 76\% two steps, 95:5 $\square: \square$ ). IR (thin film) 1747, 1722, 1678, 1514,1226, 1036 $\mathrm{cm}^{-1} ;{ }^{1} \mathrm{H}$ NMR $\left(400 \mathrm{MHz}, \mathrm{CDCl}_{3}\right) \square 8.79(\mathrm{~s}, 1 \mathrm{H}), 7.37-7.27(\mathrm{~m}, 10 \mathrm{H}), 6.60(\mathrm{~d}, J=8.5 \mathrm{~Hz}$, $1 \mathrm{H}), 6.49(\mathrm{~d}, J=3.5 \mathrm{~Hz}, 1 \mathrm{H}), 5.41(\square \mathrm{t}, J=9.7 \mathrm{~Hz}, 1 \mathrm{H}), 4.70-4.64(\mathrm{~m}, 2 \mathrm{H}), 4.57-4.45(\mathrm{~m}$, $3 \mathrm{H}), 4.10-4.02(\mathrm{~m}, 2 \mathrm{H}), 3.63-3.55(\mathrm{~m}, 2 \mathrm{H}), 1.97(\mathrm{~s}, 3 \mathrm{H}) ;{ }^{13} \mathrm{C} \mathrm{NMR}\left(400 \mathrm{MHz}, \mathrm{CDCl}_{3}\right) \square$ $169.3,161.8,159.9,137.6,137.0,128.8,128.4,128.4,128.3,128.1,127.8,94.3,92.1$, 90.7, 76.0, 73.6, 73.0, 72.1, 69.9, 68.4, 53.5, 20.9; ESI MS $m / z\left(\mathrm{M}^{+}+\mathrm{Na}^{+}\right)$calcd 710.9763 , found 710.9762 .

tert-Butyldimethylsilyl 3-O - acet y l-2-O - be n z y l-4,6-O-benzylidene- $\square-D-$ mannopyranosyl-(1 $\square$ 4)-3,6-di- $O$-benzyl-2-deoxy-2-trichloroacetimido- $\square-D-$

glucopyranoside 8. Phenyl sulfoxide $7(1.50 \mathrm{~g}, 2.56 \mathrm{mmol})$ and 6 (2.53 g, $4.09 \mathrm{mmol})$ were coevaporated separately (important!) with toluene $(3 \times 10 \mathrm{~mL})$ and dried under vacuum overnight. Sulfoxide 7 was dissolved in $\mathrm{CH}_{2} \mathrm{Cl}_{2}(26 \mathrm{~mL})$ and cooled to $-78^{\circ} \mathrm{C}$. Di-t-butyl pyridine $(1.15 \mathrm{~mL}, 5.12 \mathrm{mmol})$ was added to the cooled solution and stirred for 10 min. Triflic anhydride (474 $\square \mathrm{L}, 2.82 \mathrm{mmol}$ ) was added and the mixture was stirred for 5 min, during which time the colorless mixture turned orange. A solution of 6 in $\mathrm{CH}_{2} \mathrm{Cl}_{2}(10 \mathrm{~mL})$ was slowly added to the reaction mixture via cannula and the reaction was stirred at $-78^{\circ} \mathrm{C}$ for $1 \mathrm{~h}$. The reaction was quenched with saturated $\mathrm{NaHCO}_{3}(20 \mathrm{~mL})$ 
and diluted with $\mathrm{CH}_{2} \mathrm{Cl}_{2}(30 \mathrm{~mL})$. The organic layer was were washed with $\mathrm{NaHCO}_{3}(2 \mathrm{x}$ $30 \mathrm{~mL}$ ), dried over $\mathrm{Na}_{2} \mathrm{SO}_{4}$, filtered, and solvents removed in vacuo. Purification by flash silica column chromatography (100\% toluene $\square 25 \%$ EtOAc/toluene) afforded tertbutyldimethylsilyl 2-O-benzyl-4,6-O-benzylidene-3-O-p-methoxybenzyl- $\square-\mathrm{D}-$ mannopyranosyl-(1\ 4)-3,6-di-O-benzyl-2-deoxy-2-trichloroacetimido- $\square-\mathrm{D}$ -

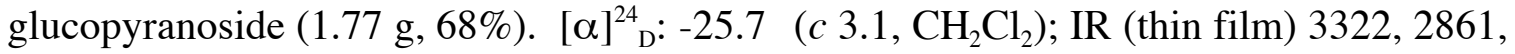
1691, 1531, $1089 \mathrm{~cm}^{-1} ;{ }^{1} \mathrm{H}$ NMR (400 MHz, $\left.\mathrm{CDCl}_{3}\right) \square 7.51-7.18(\mathrm{~m}, 22 \mathrm{H}), 6.97$ (d, $J=$ $7.5 \mathrm{~Hz}, 1 \mathrm{H}), 6.87-6.85(\mathrm{~m}, 2 \mathrm{H}), 5.54(\mathrm{~s}, 1 \mathrm{H}), 5.19(\mathrm{~d}, J=7.7 \mathrm{~Hz}, 1 \mathrm{H}), 5.09(\mathrm{~d}, J=10.4$ $\mathrm{Hz}, 1 \mathrm{H}), 4.85(\mathrm{~d}, J=2.6 \mathrm{~Hz}, 2 \mathrm{H}), 4.68-4.45(\mathrm{~m}, 6 \mathrm{H}), 4.14-4.06(\mathrm{~m}, 3 \mathrm{H}), 3.99(\square \mathrm{t}, J=8.7$ $\mathrm{Hz}, 1 \mathrm{H}), 3.80(\mathrm{~s}, 3 \mathrm{H})$ 3.79-3.77 (m, 1H), 3.68-3.39 (m, 6H), 3.16-3.15 (m, 1H), 2.37 (s, 1H), 0.90 (s, 9H), 0.15 (s, 3H), 0.12 (s, 3H); ${ }^{13} \mathrm{C}$ NMR (400 MHz, $\left.\mathrm{CDCl}_{3}\right) \square$ 161.8, 159.3, $138.7,138.6,138.0,137.8,130.7,129.3,129.2,129.1,128.7,128.6,128.4,128.4,128.4$, $128.3,128.1,127.9,127.8,126.3,125.5,113.9,101.9,101.5,94.4,92.7,78.8,78.3,78.0$, $77.4,77.3,75.2,75.1,74.8,73.8,72.5,68.9,68.7,67.6,60.7,55.5,25.9,18.1,-4.0,-4.9$; ESI MS $m / z\left(\mathrm{M}^{+}+\mathrm{Na}^{+}\right)$calcd 1100.3312 , found 1100.3278 .

To a stirring solution of tert-butyldimethylsilyl 2-O-benzyl-4,6- $O$-benzylidene-3- $O$ - $p$ methoxybenzyl- $\square-D-m a n n o p y r a n o s y l-(1 \square$ 4)-3,6-di-O-benzyl-2-deoxy-2trichloroacetimido- $\mathrm{C}$-D-glucopyranoside $(1.0 \mathrm{~g}, 0.93 \mathrm{mmol})$ in $\mathrm{CH}_{2} \mathrm{Cl}_{2}(4.5 \mathrm{~mL})$ and $\mathrm{H}_{2} \mathrm{O}$ (0.5 mL) was added 2,3-dichloro-5,6-dicyanobenzoquinone (252 mg, $1.11 \mathrm{mmol})$. After stirring for $45 \mathrm{~min}$, the reaction mixture was diluted with $\mathrm{CH}_{2} \mathrm{Cl}_{2}(45 \mathrm{~mL})$. The organic layer was washed with $\mathrm{NaHCO}_{3}(2 \times 25 \mathrm{~mL}), \mathrm{H}_{2} \mathrm{O}(1 \times 25 \mathrm{~mL})$, dried over $\mathrm{Na}_{2} \mathrm{SO}_{3}$, filtered and solvents removed in vacuo. Purification by flash silica column chromatography (20\% EtOAc/hexanes) gave a white solid. The solid (743 mg, 0.77 
mmol) was dissolved in $\mathrm{CH}_{2} \mathrm{Cl}_{2}(8 \mathrm{~mL})$ and cooled to $0^{\circ} \mathrm{C}$. Acetic anhydride $(147 \square \mathrm{L}$, $1.55 \mathrm{mmol}$ ) was added and stirred for $5 \mathrm{~min}$. Dimethylaminopyridine (114 $\mathrm{mg}, 0.93$ mmol) was added and the stirring mixture was warmed slowly to room temperature over 2 h. The reaction was diluted with $\mathrm{CH}_{2} \mathrm{Cl}_{2}(15 \mathrm{~mL})$ and washed with $5 \% \mathrm{HCl}(2 \times 15$ $\mathrm{mL}), \mathrm{H}_{2} \mathrm{O}(1 \times 15 \mathrm{~mL}), \mathrm{NaHCO}_{3}(2 \times 15 \mathrm{~mL})$. The organic layer was dried over $\mathrm{Na}_{2} \mathrm{SO}_{4}$, filtered, and solvents removed in vacuo. Purification by flash silica column

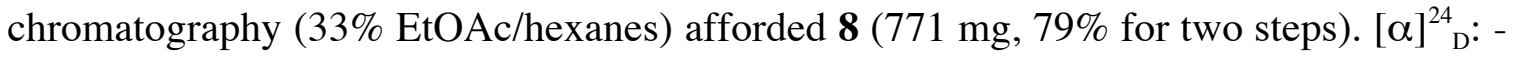
$45.0^{\circ}\left(c=0.7, \mathrm{CH}_{2} \mathrm{Cl}_{2}\right)$; IR (thin film) $3339,2858,1738,1691,1092,1068 \mathrm{~cm}^{-1} ;{ }^{1} \mathrm{H} \mathrm{NMR}$ $\left(400 \mathrm{MHz}, \mathrm{CDCl}_{3}\right) \square 7.47-7.28(\mathrm{~m}, 20 \mathrm{H}), 6.99(\mathrm{~d}, J=7.5 \mathrm{~Hz}, 1 \mathrm{H}), 5.48(\mathrm{~s}, 1 \mathrm{H}), 5.22(\mathrm{~d}$, $J=7.7 \mathrm{~Hz}, 1 \mathrm{H}), 5.11(\mathrm{~d}, J=10.4 \mathrm{~Hz}, 1 \mathrm{H}), 4.87-4.82(\mathrm{~m}, 2 \mathrm{H}), 4.74-4.50(\mathrm{~m}, 5 \mathrm{H}), 4.18-$ $4.02(\mathrm{~m}, 5 \mathrm{H}), 3.71(\mathrm{dq}, J=2.3,5.6 \mathrm{~Hz}, 2 \mathrm{H}), 3.57-3.52(\mathrm{~m}, 2 \mathrm{H}), 3.41-3.38(\mathrm{~m}, 1 \mathrm{H}), 3.23-$ $3.22(\mathrm{~m}, 1 \mathrm{H}), 1.99(\mathrm{~s}, 3 \mathrm{H}), 0.92(\mathrm{~s}, 9 \mathrm{H}), 0.16(\mathrm{~s}, 3 \mathrm{H}), 0.13(\mathrm{~s}, 3 \mathrm{H}) ;{ }^{13} \mathrm{C}$ NMR $(400 \mathrm{MHz}$, $\left.\mathrm{CDCl}_{3}\right) \square 170.6,161.8,138.7,138.2,137.9,137.4,129.3,128.8,128.5,128.4,128.4$, $128.4,128.2,128.2,128.0,128.0,127.8,126.4,101.9,101.3,94.4,92.7,78.1,77.3,76.5$, $75.9,75.7,75.1,74.8,73.8,72.6,68.8,68.6,67.5,60.8,25.8,21.22,18.1,-4.0,-4.9 ;$ ESI MS m/z $\left(\mathrm{M}^{+}+\mathrm{Na}^{+}\right)$calcd 1022.2843, found 1022.2807.

tert-Butyldimethylsilyl 3,6-di-O-acetyl-2,4-di-O-benzyl- $-D$-mannopyranosyl-(1] 4)3,6-di- $O$-benzyl-2-deoxy-2-trichloroacetimido- $\square$-D-glucopyranoside 9. To a solution of $8(213 \mathrm{mg}, 0.213 \mathrm{mmol})$ in $\mathrm{CH}_{2} \mathrm{Cl}_{2}(2 \mathrm{~mL})$ were added freshly dried $4 \AA$ molecular sieves $(750 \mathrm{mg})$. After stirring for $1 \mathrm{~h}$, the mixture was cooled to $-78^{\circ} \mathrm{C}$. Triethylsilane (102 $\square \mathrm{L}, 0.64 \mathrm{mmol}$ ) was added and the resulting solution was stirred for $5 \mathrm{~min}$. Dichlorophenylborane (83 $\square \mathrm{L}, 0.64 \mathrm{mmol}$ ) was added and the mixture was stirred for 30 min at $-78^{\circ} \mathrm{C}$. The reaction was quenched with the addition of triethylamine $(0.4 \mathrm{~mL})$ 
and methanol $(0.4 \mathrm{~mL})$ and diluted with $\mathrm{CH}_{2} \mathrm{Cl}_{2}(20 \mathrm{~mL})$. The organic layer was washed with $\mathrm{NaHCO}_{3}(2 \times 20 \mathrm{~mL}), \mathrm{H}_{2} \mathrm{O}(1 \times 20 \mathrm{~mL})$, dried over $\mathrm{Na}_{2} \mathrm{SO}_{4}$, filtered, and solvents removed in vacuo. Purification by flash silica column chromatography (25\% EtOAc/hexanes) afforded a white solid. The solid (170 mg, $0.17 \mathrm{mmol})$ was dissolved in $\mathrm{CH}_{2} \mathrm{Cl}_{2}(2 \mathrm{~mL})$ and cooled to $0^{\circ} \mathrm{C}$. Acetic anhydride $(32 \square \mathrm{l}, 0.34 \mathrm{mmol})$ was added and stirred for $5 \mathrm{~min}$. Dimethylaminopyridine $(25 \mathrm{mg}, 0.20 \mathrm{mmol})$ was added and the stirring mixture was warmed slowly to room temperature over $2.5 \mathrm{~h}$. The reaction mixture was then diluted with $\mathrm{CH}_{2} \mathrm{Cl}_{2}(15 \mathrm{~mL})$ and washed with $5 \% \mathrm{HCl}(2 \times 15 \mathrm{~mL}), \mathrm{H}_{2} \mathrm{O}(1 \times 15$ $\mathrm{mL})$, and $\mathrm{NaHCO}_{3}(2 \times 15 \mathrm{~mL})$. The organic layer was dried over $\mathrm{Na}_{2} \mathrm{SO}_{4}$, filtered, and the solvents removed in vacuo. Purification by flash silica column chromatography $(25 \%$

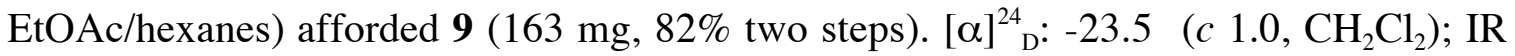
(thin film) 3342, 2858, 1742, 1691, 1234, $1073 \mathrm{~cm}^{-1} ;{ }^{1} \mathrm{H}$ NMR (400 MHz, $\left.\mathrm{CDCl}_{3}\right) \square 7.39$ $7.22(\mathrm{~m}, 20 \mathrm{H}), 6.93(\mathrm{~d}, J=7.5 \mathrm{~Hz}, 1 \mathrm{H}), 5.15-5.11(\mathrm{~m}, 2 \mathrm{H}), 4.86-4.49(\mathrm{~m}, 9 \mathrm{H}), 4.25-4.08$ (m, 4H), $3.94(\mathrm{~d}, J=3.0 \mathrm{~Hz}, 1 \mathrm{H}), 3.85(\square \mathrm{t}, J=9.7 \mathrm{~Hz}, 1 \mathrm{H}), 3.74(\mathrm{dd}, J=2.7,11.1 \mathrm{~Hz}$, 1H), $3.66(\mathrm{dd}, J=3.4,11.1 \mathrm{~Hz}, 1 \mathrm{H}), 3.54-3.37(\mathrm{~m}, 3 \mathrm{H}), 1.93(\mathrm{~s}, 3 \mathrm{H}), 1.89(\mathrm{~s}, 3 \mathrm{H}), 0.89$ (s, 9H), 0.14 (s, 3H), $0.10(\mathrm{~s}, 3 \mathrm{H}) ;{ }^{13} \mathrm{C} \mathrm{NMR}\left(400 \mathrm{MHz}, \mathrm{CDCl}_{3}\right) \square 171.0,170.4,161.8$, $139.0,138.5,138.0,128.7,128.7,128.5,128.4,128.1,128.1,127.9,127.9,127.6,100.8$, $94.5,92.7,77.9,77.7,77.4,76.2,75.9,75.1,74.9,74.3,73.8,73.6,73.3,68.9,63.6,60.4$, 25.8, 21.2, 20.9, 18.1, -4.0 -4.9; ESI MS m/z $\left(\mathrm{M}^{+}+\mathrm{Na}^{+}\right)$calcd 1066.3105, found 1066.3124.

\section{3,6-Di- $O$-acetyl-2,4-di- $O$-benzyl- $-\mathrm{D}-\mathrm{mannopyranosyl-(1]} 4$ )-3,6-di-O-benzyl-2-} deoxy-2-trichloroacetimido- $\square$-D-glucopyranosyl trichloroacetimidate 4 . A solution of $9(330 \mathrm{~g}, 0.31 \mathrm{mmol})$ in THF $(3 \mathrm{~mL})$ was cooled to $0^{\circ} \mathrm{C}$. Acetic acid $(27 \square \mathrm{L}, 0.47$ 
$\mathrm{mmol})$ and then tetrabutylammonium fluoride (1.0 M in THF, $470 \square \mathrm{L}, 0.47 \mathrm{mmol})$ were added to the cooled solution. The reaction mixture was allowed to warm slowly to room temperature while stirring for $3 \mathrm{~h}$. The reaction mixture was diluted with EtOAc and washed with $\mathrm{NaHCO}_{3}(2 \times 10 \mathrm{~mL})$ and $\mathrm{H}_{2} \mathrm{O}(1 \times 10 \mathrm{~mL})$. The organic layer was then dried over $\mathrm{Na}_{2} \mathrm{SO}_{4}$, filtered, and solvents removed in vacuo. The crude material (294 mg, $0.31 \mathrm{mmol})$ was dissolved in $\mathrm{CH}_{2} \mathrm{Cl}_{2}(3 \mathrm{~mL})$ and trichloroacetonitrile $(0.3 \mathrm{~mL})$ and cooled to $0^{\circ} \mathrm{C}$. After stirring for $5 \mathrm{~min}$, DBU $(9.3 \square \mathrm{L}, 0.062 \mathrm{mmol})$ was added and the reaction mixture was allowed to stir for $1.5 \mathrm{~h}$. The reaction mixture was passed through a silica plug, washed with EtOAc and the solvents removed in vacuo. Purification by flash silica column chromatography (25\% EtOAc/hexanes) afforded 4 (298 mg, 89\%, 95:5

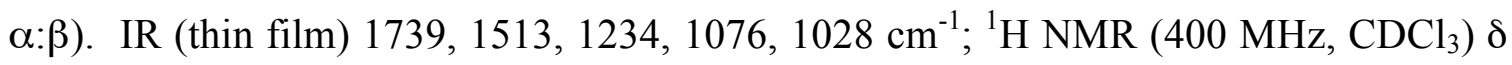
$8.71(\mathrm{~s}, 1 \mathrm{H}), 7.43-7.26(\mathrm{~m}, 20 \mathrm{H}), 6.52(\mathrm{~d}, J=3.3 \mathrm{~Hz}, 1 \mathrm{H}), 6.46(\mathrm{~d}, J=7.6 \mathrm{~Hz}, 1 \mathrm{H}), 5.02$ (d, $J=11.9 \mathrm{~Hz}, 1 \mathrm{H}), 4.87(\mathrm{~d}, J=12.1 \mathrm{~Hz}, 1 \mathrm{H}), 4.77-4.66(\mathrm{~m}, 6 \mathrm{H}), 4.59-4.50(\mathrm{~m}, 2 \mathrm{H})$, 4.30-4.22 (m, 4H), 3.95-3.86 (m, 4H), $3.70(\mathrm{~s}, 2 \mathrm{H}), 3.38-3.35(\mathrm{~m}, 1 \mathrm{H}), 1.97(\mathrm{~s}, 3 \mathrm{H}), 1.91$ (s, 3H); ${ }^{13} \mathrm{C}$ NMR $\left(400 \mathrm{MHz}, \mathrm{CDCl}_{3}\right) \square 170.9,170.3,162.0,160.1,138.4,138.4,137.9$, $137.5,128.8,128.7,128.7,128.6,128.6,128.4,128.4,128.4,128.3,128.3,128.2,128.1$, $128.1,128.0,127.9,127.9,127.9,127.8,100.6,94.5,92.1,91.0,76.7,76.2,75.8,75.7$, 75.0, 74.8, 73.9, 73.8, 73.7, 73.4, 73.1, 67.9, 63.1, 54.1, 21.1, 20.8; ESI MS m/z $\left(\mathrm{M}^{+}+\right.$ $\mathrm{Na}^{+}$) calcd 1095.1336, found 1095.1343.

$n$-Pentenyl 2- $O$-acetyl-3,4,6-tri-O-benzyl-[-D-mannopyranosyl-(1[ 3)-[2-O-acetyl3,4,6-tri-O-benzyl-[-D-mannopyranosyl-(1] 6)]-2,4-di-O-benzyl-[-Dmannopyranosyl-(1 $\square$ 4)-3,6-di-O-benzyl-2-deoxy-2-trichloroacetimido-[-Dglucopyranosyl-(1 $\square$ 4)-3,6-di- $O$-benzyl-2-deoxy-2-trichloroacetimido- $\square-D-$ 
glucopyranoside 1. Glycosylated resin from automated synthesis was dried in vacuo for $18 \mathrm{~h}$ over phosphorous pentoxide and transferred into a solid-phase round bottom flask with glass frit. The resin was swelled with $5 \mathrm{ml} \mathrm{CH}_{2} \mathrm{Cl}_{2}$, purged with an atmosphere of ethylene followed by the addition of 10 mol \% Grubbs' catalyst (bis(tricyclohexylphosphine)benzylidene ruthenium (IV) dichloride. The reaction mixture was stirred for $24 \mathrm{~h}$ under an atmosphere of ethylene, an additional $10 \mathrm{~mol} \%$ Grubbs' catalyst was added, and the reaction was allowed to stir an additional $24 \mathrm{~h}$ under an atmosphere of ethylene. Triethylamine (100 equiv.) and tris hydroxymethylphosphine (50 equiv.) were added, and the mixture stirred $2 \mathrm{~h}$ at room temperature. The reaction was diluted in $\mathrm{CH}_{2} \mathrm{Cl}_{2}$ and washed 3 times with water. The aqueous fractions were washed with additional $\mathrm{CH}_{2} \mathrm{Cl}_{2}$. The organic fractions were combined, dried over $\mathrm{MgSO}_{4}$, filtered, and dried to yield adark oil.

The crude product was analyzed by HPLC (Waters Nova-pak ${ }^{\square}$ silica column (3.9 $\mathrm{x} 150 \mathrm{~mm}$ ) with EtOAc/hexanes as the mobile phase), monitoring at $260 \mathrm{~nm}$. A portion of the crude product was purified by semi-preparative HPLC using a Waters prep Novapak $^{\square}$ silica column $(7.8 \times 300 \mathrm{~mm})$ with a gradient of EtOAc/hexanes. Semi-preparative HPLC yielded $3 \mathrm{mg}$ of $\mathbf{1}$ that corresponded to $\mathbf{1}$ made by solution phase synthesis. IR (thin film) 2867, 1746, 1711, 1693, 1235, $1077 \mathrm{~cm}^{-1} ;{ }^{1} \mathrm{H}$ NMR (400 MHz, $\mathrm{CDCl}_{3}$ ) $\square 7.41-$ $7.08(\mathrm{~m}, 60 \mathrm{H}), 6.91(\mathrm{~d}, J=7.8 \mathrm{~Hz}, 1 \mathrm{H}), 6.40(\mathrm{~d}, J=7.9 \mathrm{~Hz}, 1 \mathrm{H}), 5.80-5.72(\mathrm{~m}, 1 \mathrm{H})$, 5.48-5.46 (m, 1H), 5.37-5.35 (m, 1H), 5.14-4.27 (m, 31H), 4.42-3.14 (m, 30H), 2.14-2.06 (m, 5H), 1.98 (s, 3H), 1.68-1.63 (m, 2H); ${ }^{13} \mathrm{C}$ NMR (400 MHz, $\left.\mathrm{CDCl}_{3}\right) \square 170.3,170.3$, $161.9,161.8,138.9,138.8,138.7,138.7,138.6,138.5,138.5,138.2,138.2,138.2,138.0$, $137.9,128.8,128.8,128.7,128.6,128.6,128.5,128.5,128.5,128.5128 .4,128.4,128.3$, 
$128.2,128.1,128.1,128.0,127.9,127.9,127.8,127.8,127.7,127.7,127.6,127.5,127.3$, 115.1, 101.5, 99.8, 99.7, 99.0, 97.8, 92.7, 81.2, 78.5, 78.2, 78.1, 77.5, 77.2, 77.0, 75.5, 75.3, 75.2, 75.0, 75.0, 74.9, 74.5, 74.5, 74.4, 74.3, 74.2, 73.6, 73.5, 73.5, 72.5, 72.0, 71.7, 71.6, 71.5, 69.4, 69.2, 68.8, 68.6, 68.5, 66.7, 58.2, 57.4, 30.2, 29.9, 28.9, 28.9, 21.3, 21.2, 21.2; ESI MS m/z $\left(\mathrm{M}^{+}+\mathrm{Na}^{+}\right)$calcd 2369.7303, found 2369.7401 . 

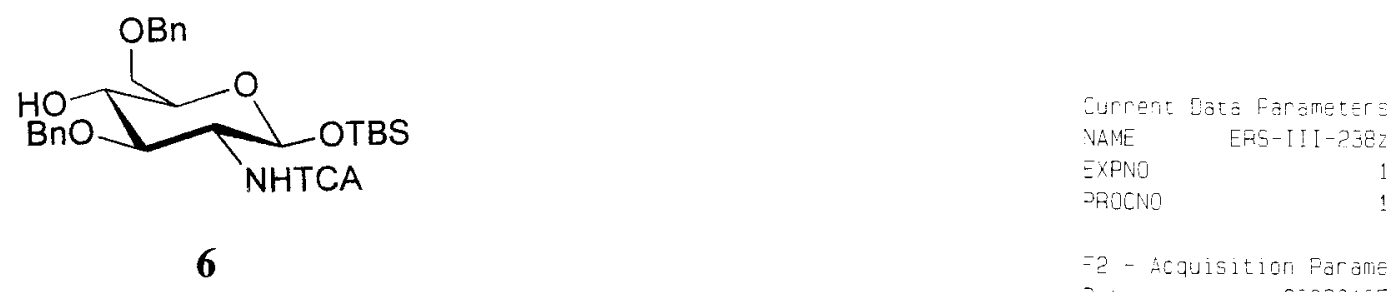

za - Acquisition Parameters Date _ 20030105

$\begin{array}{lr}\text { Date } & 20020105 \\ \text { Time } & 20.14\end{array}$

INSTRUHA Spect

PROBHO 5mm BEO 8B-1

JULPFOG 2930

TD 40062

v

J5 2

SWH $\quad 4006.410 \mathrm{~Hz}$

FIDRES $\quad 0.100005 \mathrm{~Hz}$

AQ 4. $4997878 \mathrm{sec}$

76

32
124.800 usec

6.00 usec

$3000 \mathrm{~K}$

$1.00000000 \mathrm{sec}$

21

CHANNEL F $1===========0$

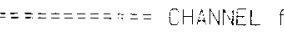

UUC: $1 \mathrm{H}$

317.90 use

$\begin{array}{lr}\text { OL1 } & 0.00 \mathrm{dE} \\ \text { SF } & 400.131800 \mathrm{MH}\end{array}$

=2 - Processing parameters

$\begin{array}{lc}\text { SI } & 32768 \\ \text { SF } & 400.1300054 \mathrm{MHz}\end{array}$
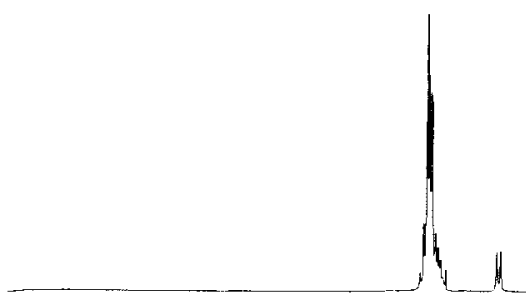

III. ate

$5 S \mathrm{~B}$

$B$

90

10 HMk piot pärāmeters

cx $\quad 20.00 \mathrm{~cm}$

$-1 \mathrm{P} \quad \mathrm{g} .453 \mathrm{FEm}$

$\begin{array}{lr}-1 & 3798.43 \mathrm{~Hz} \\ -20 & -0.520 \mathrm{FHH}\end{array}$

$=5 \quad-20.96 \mathrm{~Hz}$

sPMand 0 Goned ormion

$42 \mathrm{CM} \quad 200.32053 \mathrm{~Hz} / \mathrm{Cm}$ 


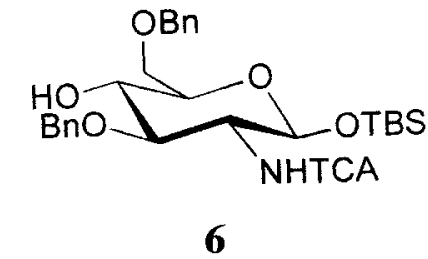

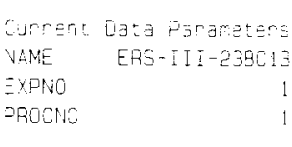

z- acauisition Faraneter

Jate_ 20030105

Time 2i.15

spect
INSTRLMM
PROEH

PULPROS

To 65536

SOLVENT CDC13

VS

SWH 25125. E29 Hz

FIDRES $\quad 0.383387 \mathrm{~Hz}$

AQ $\quad 1.3042164 \mathrm{sec}$

गW 145065

JE

TE $\quad 3.000 \mathrm{~K}$

$01 \quad 2.00000000 \mathrm{sec}$

$0.03000000 \mathrm{sec}$

0.00002000595

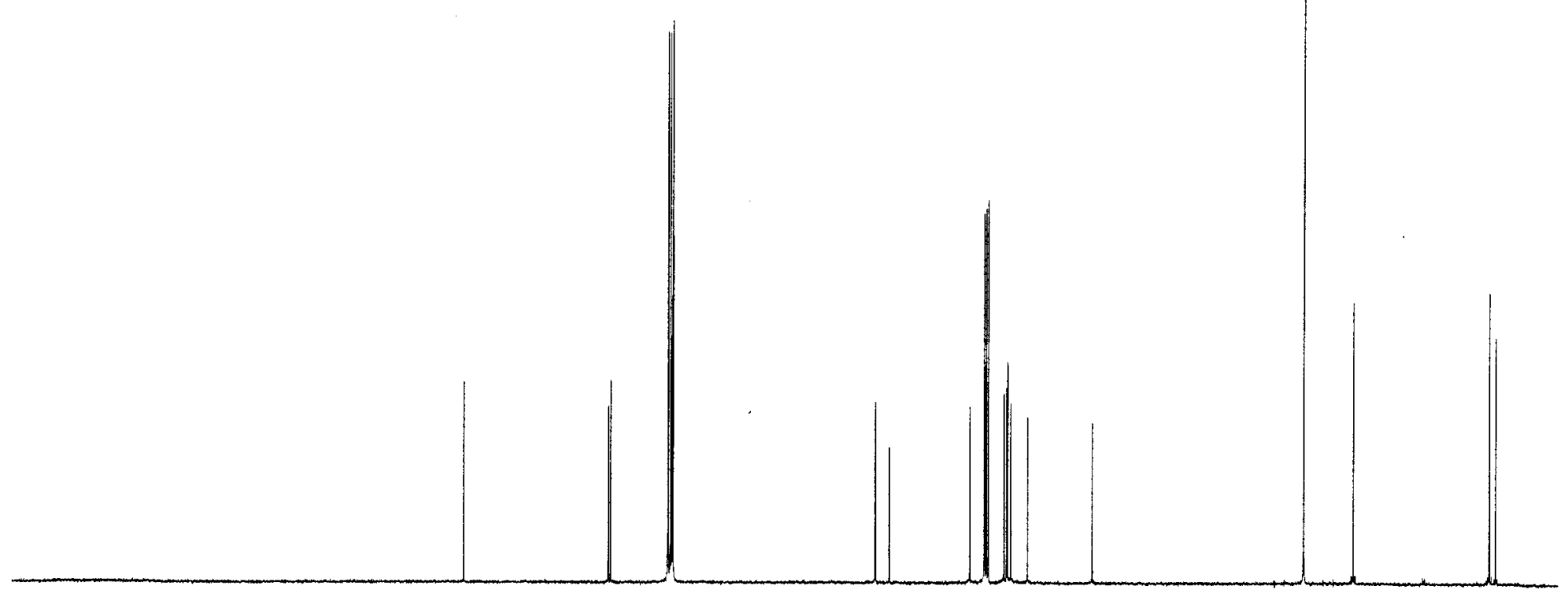

$==x=z=x== \pm=$ CHANINEL

vict

ग1

$5 F O$

15.25 use

$100.6237959 \mathrm{MH}$

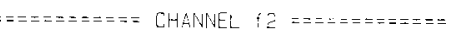

SPEPRE?

VUC2 Wa $1 \mathrm{H}$

$\begin{array}{ll}\text { NUC2 } & 1 \mathrm{H} \\ \text { SCFD? } & 107.50 \mathrm{usec}\end{array}$

2412

$0.00 \mathrm{de}$

24. $00 \mathrm{~dB}$

$4001310005 \mathrm{kH}$

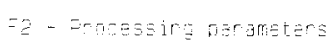

Si

SF $100.6127614 \mathrm{MHz}$

WDW $55 \mathrm{~B}$

$\begin{array}{cc}55 \mathrm{~B} & -\mathrm{B} \\ -\mathrm{B} & 1.0 \mathrm{H}\end{array}$

$-\mathrm{B}$
$\mathrm{GC}$

ID MAr piot parareters

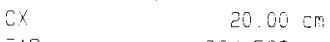

$=1 \mathrm{~F} \quad 234.536 \mathrm{pom}$

23597.30 tiz

$-15.190 \mathrm{por}$

$\begin{array}{ll}=2 & -1529.33 \mathrm{~Hz} \\ \text { JPHMM } & -248620 \mathrm{CHM}\end{array}$

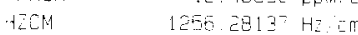




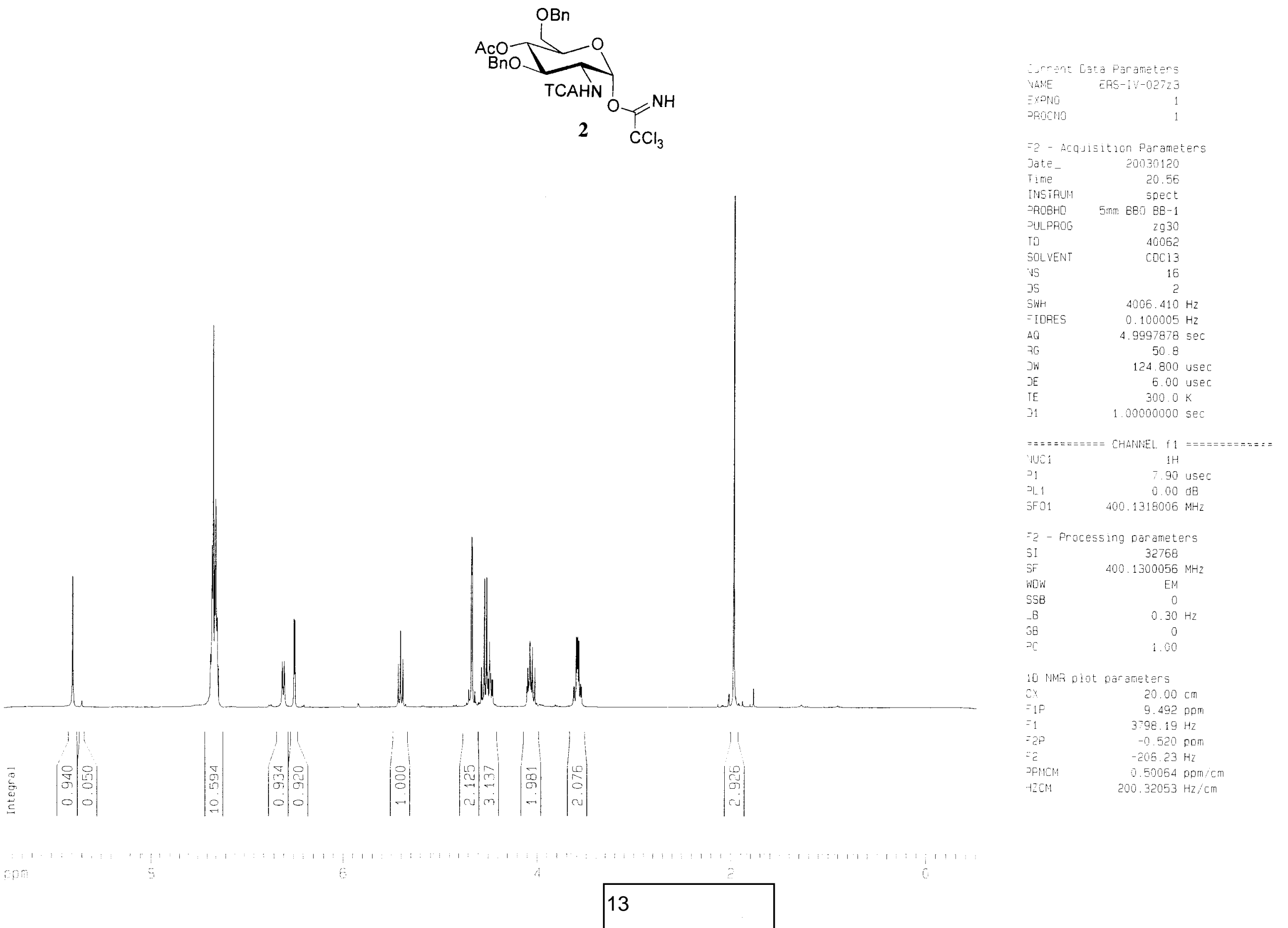




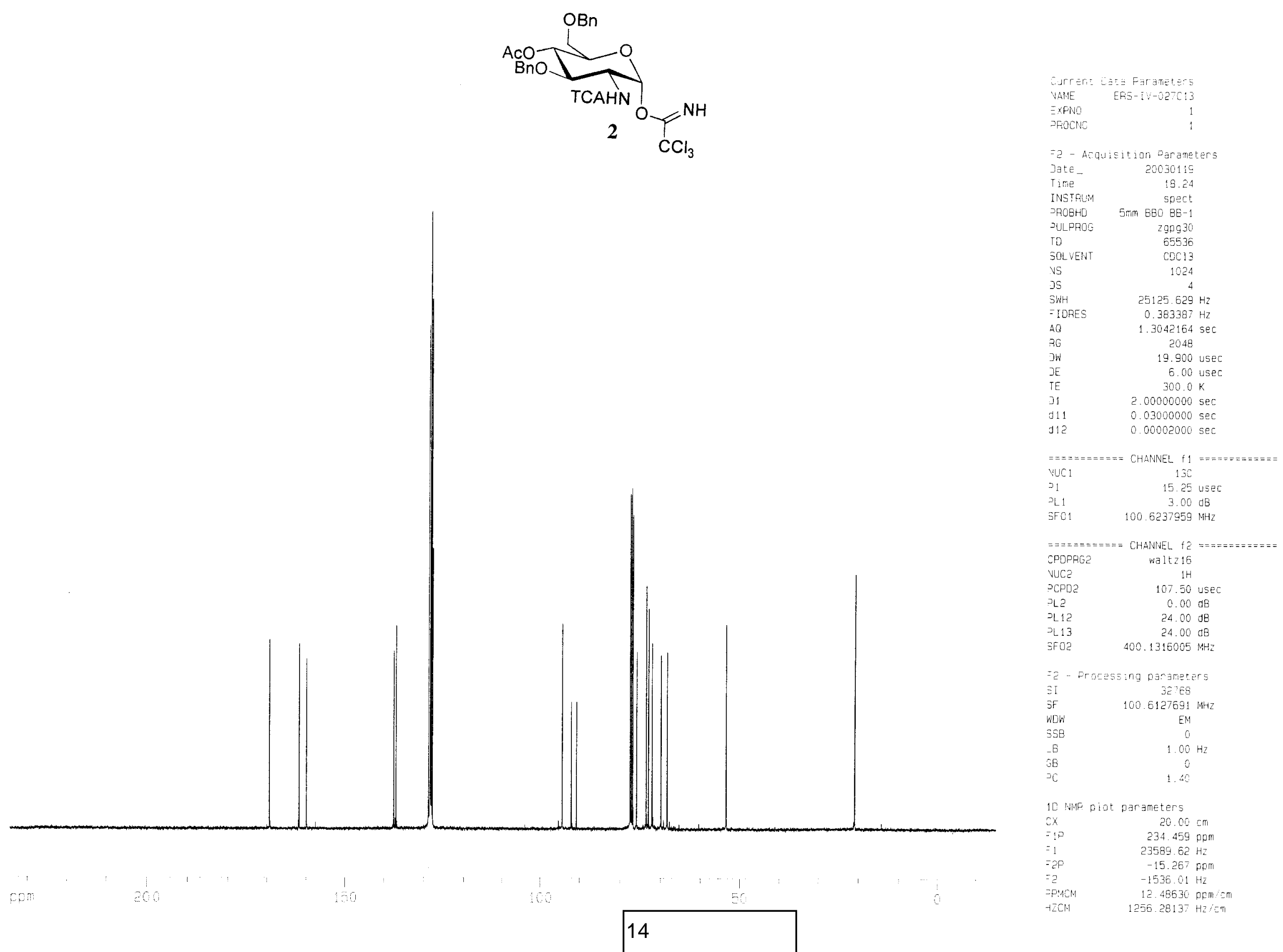




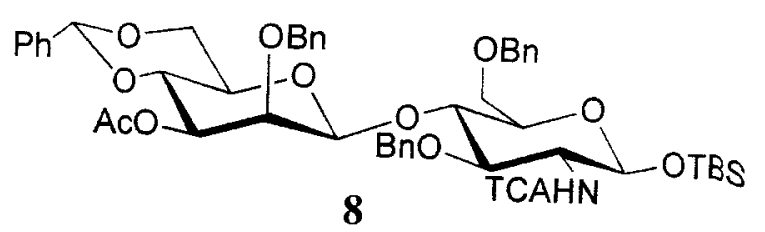

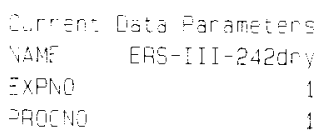

=a - Acqujisituor Farämeter

Jate 20021028

Time $\quad 15.02$

spect
INGTRLIM

YULPROO 2930

TD 40062

SOLVENT CDC13

NS

SWH $\quad \begin{gathered}2 \\ \text { SW }\end{gathered}$

$\begin{array}{ll}\text { SWH } & 4006.410 \mathrm{~Hz} \\ \text { IILRES } & 0.100005 \mathrm{~Hz}\end{array}$

AQ $\quad 4.9997878 \mathrm{sec}$

$76 \quad 80.6$

JW 124.800 usec

6.00 use

J1 $1.00000000 \mathrm{sec}$

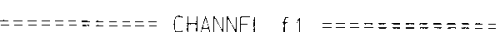

$\begin{array}{ll}\text { WUC } & 1 \mathrm{H} \\ =1 & 7.99\end{array}$

$\begin{array}{rr}\text { ZL1 } & 0.00 \mathrm{~dB} \\ \text { SFD! } & 400.1318006 \mathrm{MHz}\end{array}$

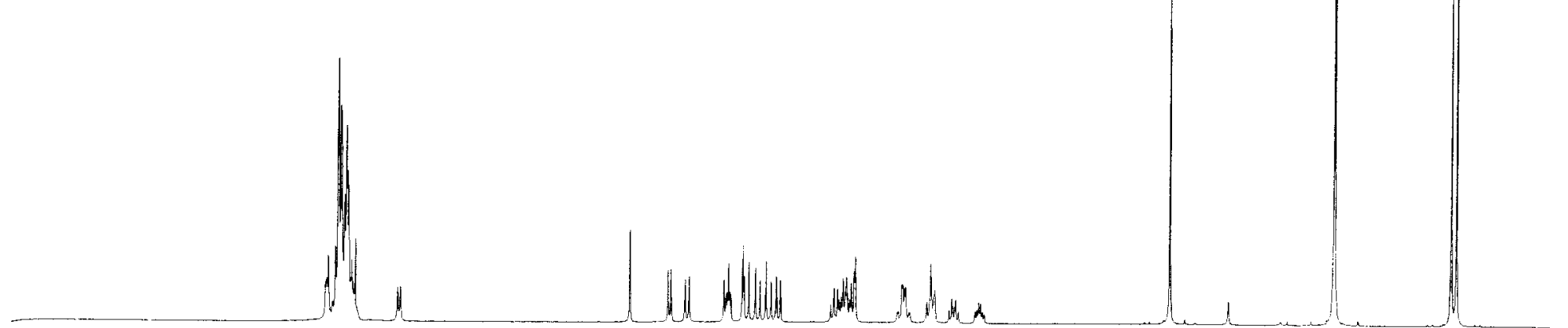

$=2$ - Processing parameters

SI $\quad 32768$

SF $\quad 400.1300060 \mathrm{MHz}$

$\begin{array}{ll}\text { WEW } & \text { EM } \\ \text { SSB } & 0\end{array}$

$\begin{array}{ll}-\mathrm{B} & 0.30 \mathrm{~Hz}\end{array}$

$68 \quad 100$

10 HMR plot parameters

$\begin{array}{ll}\mathrm{Cx} & 20.00 \mathrm{~cm} \\ =1 \mathrm{~F} & 9.491 \mathrm{cpm}\end{array}$

$=1 \quad 3797.82 \mathrm{~Hz}$

$=5 P \quad-0.521 \mathrm{opm}$

c $-2.55 .59 \mathrm{~Hz}$

o $50064 \mathrm{pom} / \mathrm{cm}$

$-17 \mathrm{CM}$

$200.32053 \mathrm{~Hz} / \mathrm{Cm}$ 

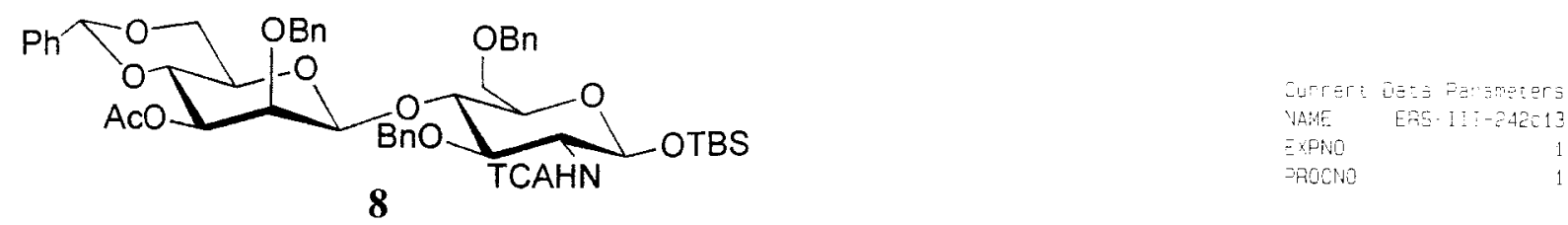

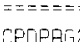

CHAMEL $+2=$

NUC?

늘

PL12

ㄴ1는

.0750 uset

107.50 usec

$0.00 \mathrm{~dB}$

$24.00 \mathrm{dE}$

$100.1316005 \mathrm{MHE}$

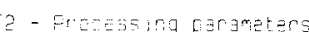

E.

If 100. El27530 MHz

WOH

-8
$2.00 \mathrm{~Hz}$

$\begin{array}{ll}68 & 6 \\ 30 & 1.40\end{array}$

10 INMR plot parameters

Cx $\quad 20.00 \mathrm{~cm}$ 234. 620 मp Q $3605.72 \mathrm{~Hz}$

$-15.106 \mathrm{per}$

$-1519.90 \mathrm{~Hz}$

12. $45630 \mathrm{ppm}=\pi$ 


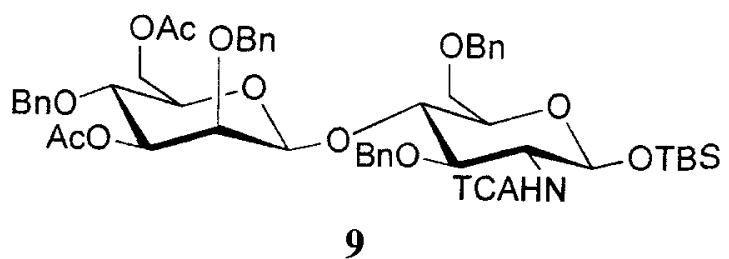

hrer: azia farateters WHE EFG-III-1ES

z- Arquisition Farameters

Jate_ 20020903

Time 21.38

INSTRUM snect

FROBHD 5 min BEO $E B-1$

$z 930$
2006

SOLVENT

75

5 int

SWDFES

A0

26

DW

TE
$\supset 1$

40062

$\operatorname{cocl} 3$

$4006.410 \mathrm{~Hz}$

$0.100005 \mathrm{~Hz}$

4. 9997878 sed

143.7

124.800 usec 5.00 usec $300.0 \mathrm{~K}$

$1.00000000 \sec$

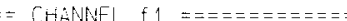

iLC

7.90 usec

L1

Fo1 $400.1318006 \mathrm{MHz}$
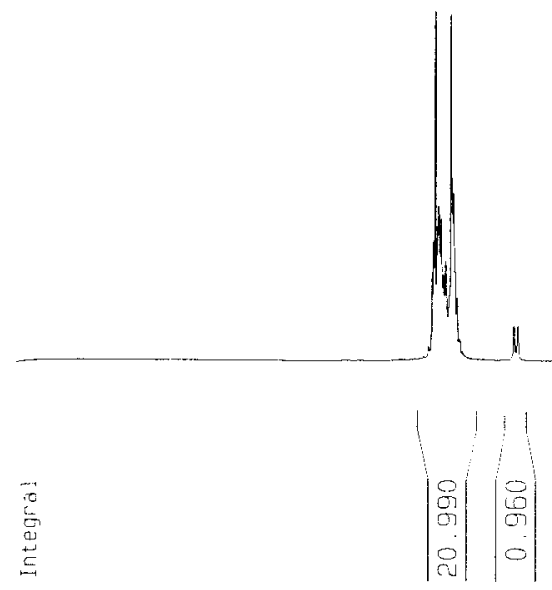

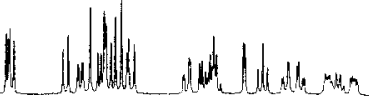

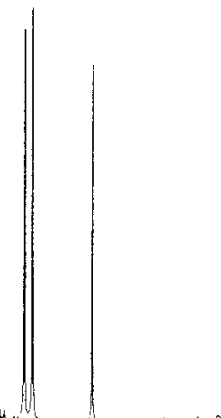

-2 - Frncessing parameters

$\begin{array}{lc}\text { Si } & 32768 \\ \text { SF } & 400.1300057 \mathrm{MHz}\end{array}$

HOW EM

$\begin{array}{lc}S S B & \mathrm{O} \\ \mathrm{B} & 0.30 \mathrm{~Hz}\end{array}$

$\mathrm{ce}$
$=\mathrm{C}$

10 MHA plot parameters

$\begin{array}{ll}c x & 20.00 \mathrm{~cm}\end{array}$

$=1 \quad 3998.06 \mathrm{~Hz}$

$-0.521 \mathrm{ppm}$

$-2.8 .35 \mathrm{~Hz}$

$0.50054 \mathrm{ppm} / \mathrm{cm}$

EFMC:

HECH

$200.32053 \mathrm{~Hz} / \mathrm{cm}$ 


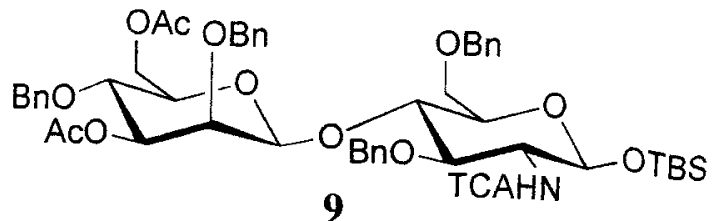

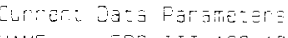

$$
\begin{aligned}
& \text { UANE ERS-III-16EC13 } \\
& \text { zenotio }
\end{aligned}
$$

-5 - Áqujaituon farameter

INS TRUM

2.11 PFO

SOLVENT

v'

JWH $\quad 25125.629 \mathrm{~Hz}$

=IDRES $\quad 0.383387 \mathrm{~Hz}$

AQ 1.3042164 se

JE

TE

$300.0 \mathrm{~K}$

$2.00000000 \mathrm{sec}$

0.03000000 5e!

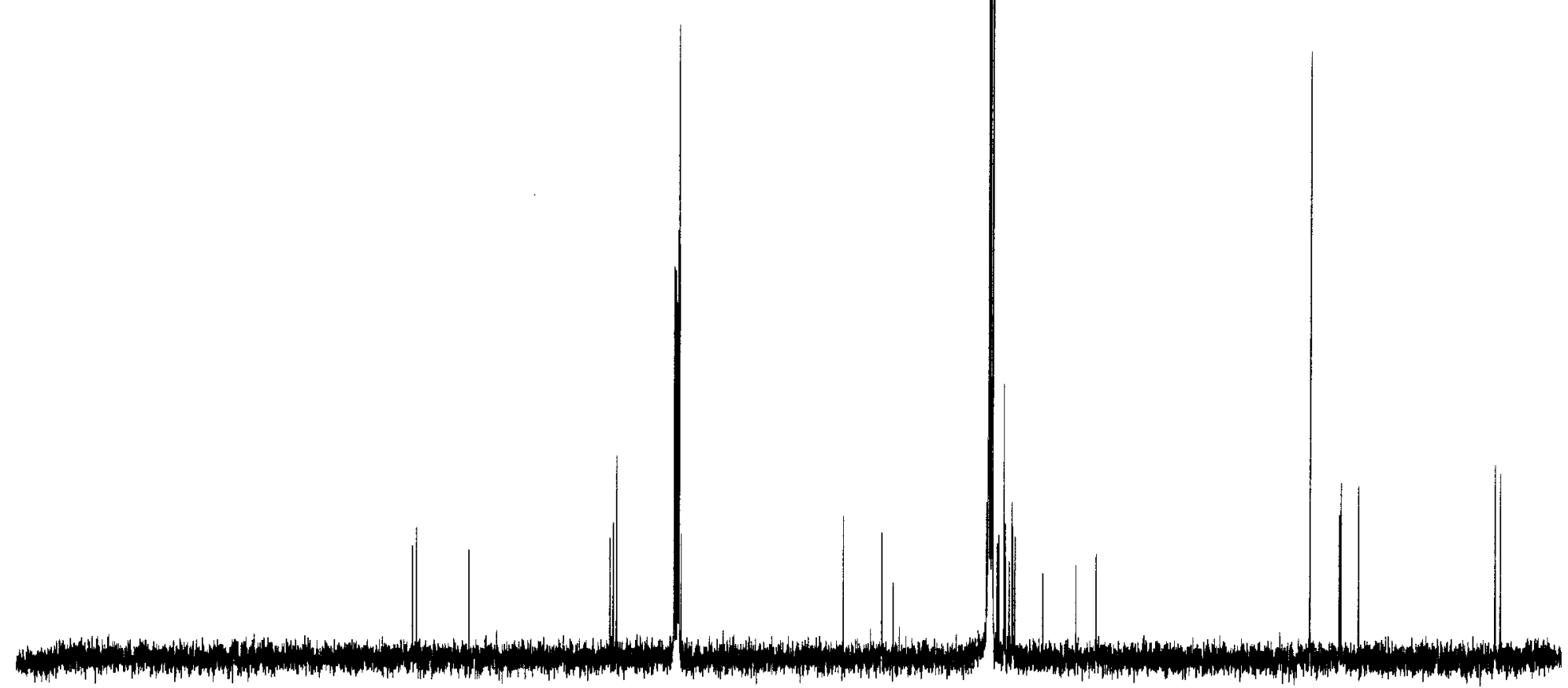

$====x===-=$ CHANNEL if

NUC1 130
I! 1
JFOL

SF01 100.6237959 MH

$== \pm=z=x=z==$ CHANHALL f

CPEPRG ? WA1t216

VUCE $1 \mathrm{H}$

JCPD2 107.50 use

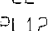

$\rightarrow \leftarrow 13$

$0.00 \mathrm{~dB}$

$24.00 \mathrm{dE}$

$400.1316005 \mathrm{MHz}$

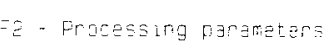

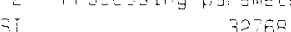

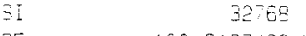

$\begin{array}{lr}\text { SF } & 100.6127492 \\ \text { WDW } & \text { EM }\end{array}$

SSE

$-B$
65

65
$=5$

\begin{tabular}{|c|c|}
\hline ¿x & $20.00 \mathrm{~cm}$ \\
\hline$=1 P$ & $234.658 \mathrm{ppm}$ \\
\hline$=1$ & $23609.56 \mathrm{~Hz}$ \\
\hline$=55$ & $-15.068 \mathrm{ppm}$ \\
\hline$=2$ & $-1516.0 \mathrm{~Hz}$ \\
\hline FPMCH & 12. $48630 \mathrm{pFm} i \mathrm{~cm}$ \\
\hline
\end{tabular}

10 ANa flat parameters 


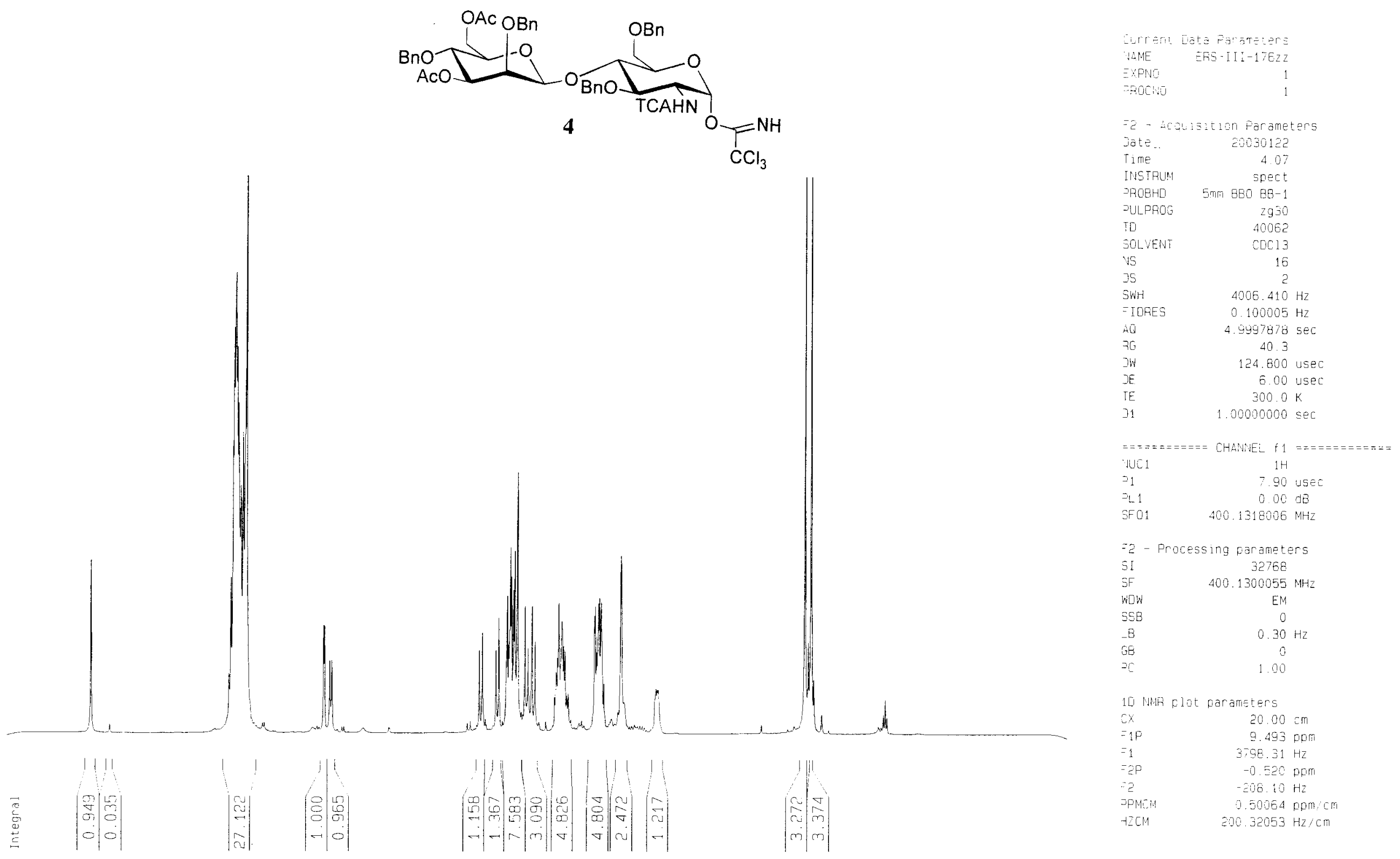

19 


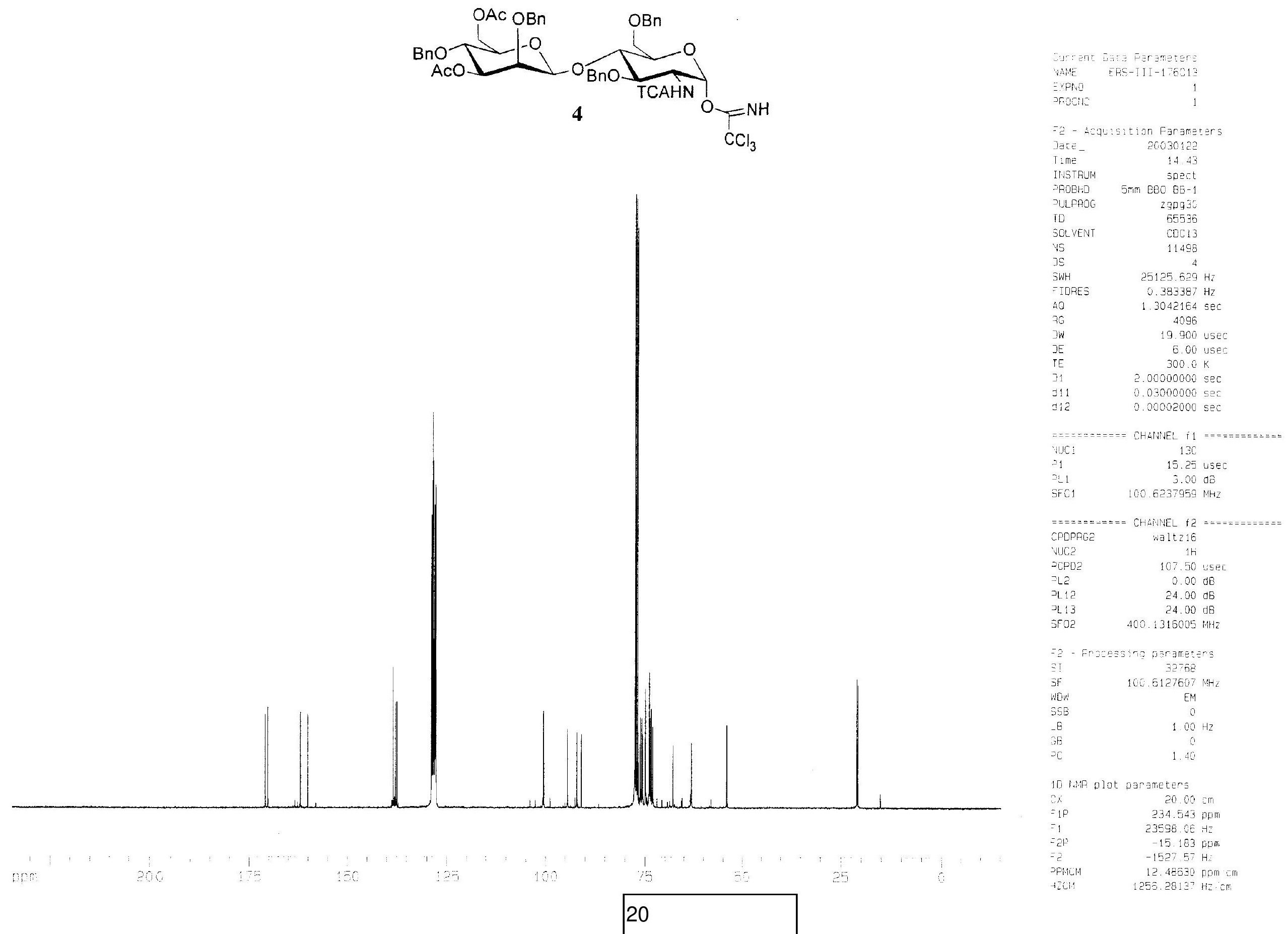




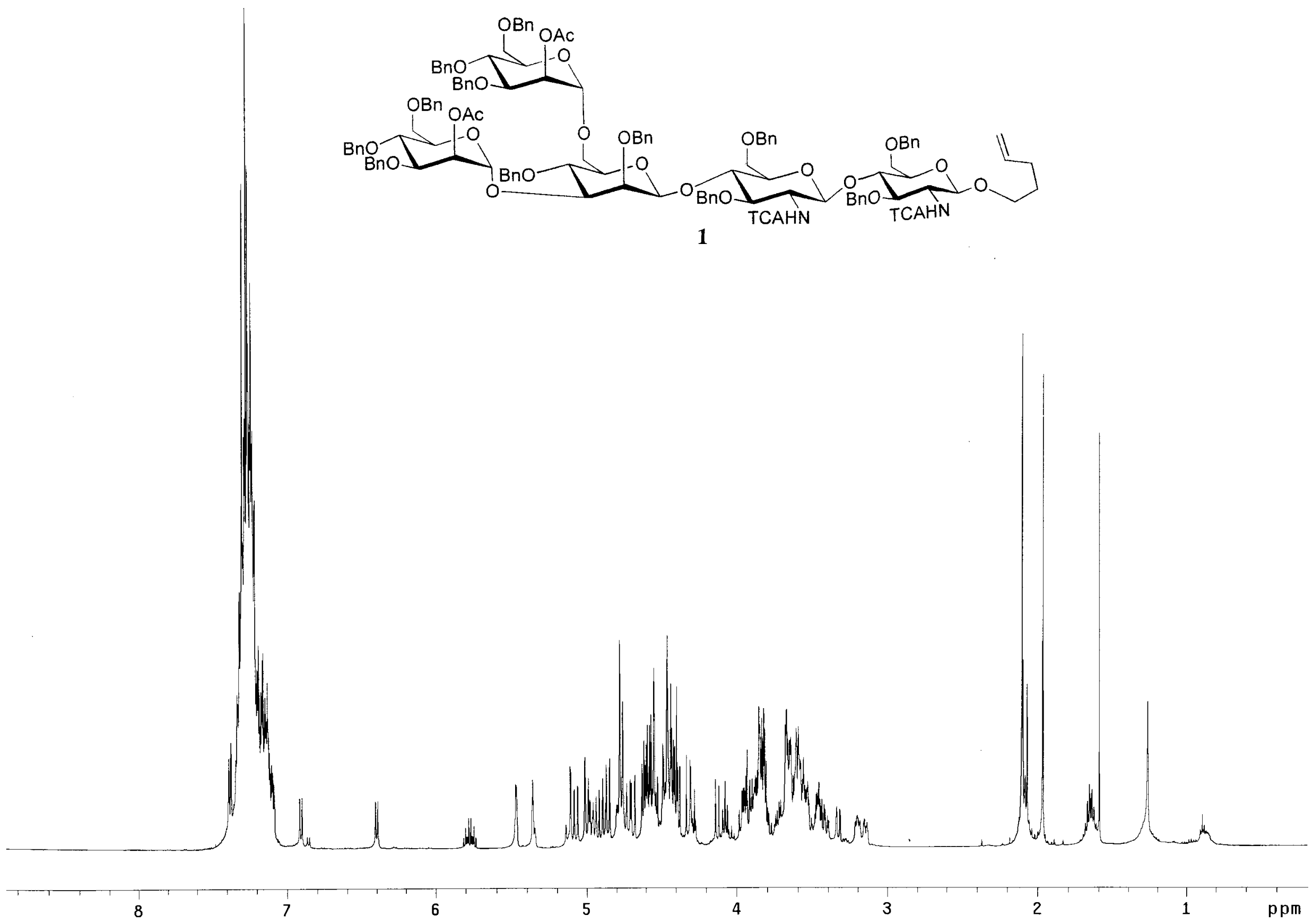

21 


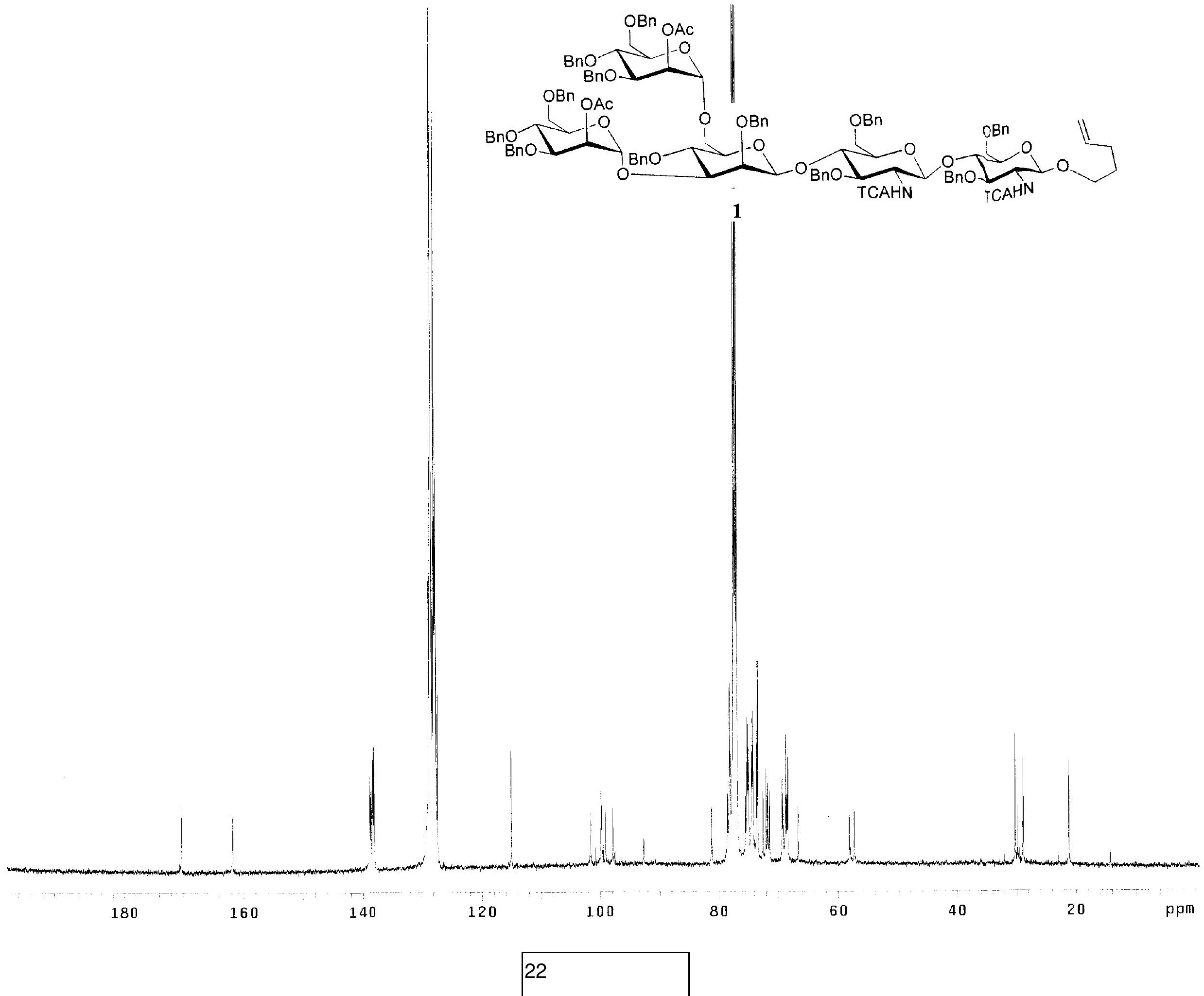

